\title{
Anapc10 orchestrates the NLRP3 inflammasome activation during cell cycle control
}

\author{
Siyu Huang ${ }^{1}$, Pin Wan ${ }^{2}$, Shanyu Huang ${ }^{1}$, Qi Xiang ${ }^{1}$, Ge Yang $^{1}$, Muhammad Adnan Shereen ${ }^{1}$, \\ Pan $\mathrm{Pan}^{2}$, Weiyong $\mathrm{Liu}^{3 * *}$, Kailang $\mathrm{Wu}^{1 * *}$, and Jianguo $\mathrm{Wu}^{1,2,4 *}$ \\ ${ }^{1}$ State Key Laboratory of Virology, College of Life Sciences, Wuhan University, Wuhan 430072 , \\ China. ${ }^{2}$ Guangdong Provincial Key Laboratory of Virology, Institute of Medical Microbiology, Jinan \\ University, Guangzhou 510632, China. ${ }^{3}$ Department of Laboratory Medicine, Tongji Hospital, \\ Tongji Medical College, Huazhong University of Science and Technology, Wuhan 430030, China. \\ ${ }^{4}$ Foshan Institute of Medical Microbiology, Foshan 528315, China.
}

*Correspondence: Jianguo Wu, State Key Laboratory of Virology and College of Life Sciences, Wuhan University, Wuhan 430072, P.R. China, Tel.: +86-27-68754979, Fax: +86-27-68754592, Email: jwu@whu.edu.cn

**Co-correspondence: Weiyong Liu,wyliu@hust.edu.cn; Kailang Wu, kailangwu@whu.edu.cn

\begin{abstract}
Activation of the NLRP3 inflammasome plays a crucial role in innate immune response. During cell division, the NLRP3 inflammasome activation must be strictly controlled. Here, we discovered the anaphase promoting complex subunit 10 (Anapc10, APC10), a substrate recognition protein of the anaphase promoting complex/cyclosome (APC/C), is a critical mediator of the NLRP3 inflammasome activation. APC10 protein interacts with NLRP3, and co-localizes with NLRP3 protein in the cytoplasm. During interphase, APC10 interacts with NLRP3 to promote the NLRP3 inflammasome activation. During mitosis, APC10 disassociates from the NLRP3 inflammasome to inhibit the inflammatory responses. This study reveals a distinct mechanism by which APC10 serves as a switch of the NLRP3 inflammasome activation during cell cycle.
\end{abstract}

Key words: Cell cycle; Cell synchronization; Pro-inflammatory cytokines; Anaphase promoting complex subunit 10, Anapc10; Anaphase-promoting complex/cyclosome, APC/C; PYRIN domain containing-3, NLRP3; The NLRP3 inflammasome

\section{Introduction}

The innate immune system is the first line of host defense which sense the signals including pathogenic microbes and cell stresses [1]. Pattern-recognition receptors (PRRs) start the innate immune system from detecting pathogen-associated molecular patterns (PAMPs) and dangerassociated molecular patterns (DAMPs) [2]. Several types of PRRs have been identified, including the Toll-like receptor (TLR), the RIG-I-like receptor (RLR), the NOD-like receptor (NLR), and the C-type lectin receptor (CLR). One of the best-characterized inflammasomes consists of the NLR family is the PYRIN domain containing-3 (NLRP3), which contains a C-terminal leucine-rich repeat (LRR), a NACHT-associated domain (NAD), and a N-terminal PYRIN domain (PYD) [3]. The LRR domain is involved in sensing ligands and auto-regulation. After the activation of NLRP3 
inflammasome, the NAD domain modified the NLRP3 oligomerization. The PYD domain of NLRP3 interacts with the PYD domain of the adaptor protein, apoptosis-associated speck-like protein with CARD domain (ASC) [4]. NLRP3 recruited ASC and pro-Caspase-1 to promote the cleavage of proCaspase-1, which in turn regulates the maturation of IL-1 $\beta$ [5]. The NLRP3 inflammasome has been reported related to several inflammatory disease named cryopyrin-associated periodic syndromes (CAPS) [6], such as Muckle-Wells syndrome, gout, and Alzheimer's disease [7,8,9].

The anaphase-promoting complex/cyclosome (APC/C) is a E3 ubiquitin ligase that controls cell cycle progression by combining specific cell cycle-related proteins, such as Securin and Cyclin B $[10,11]$. APC/C activity is strictly dependent on its co-activators: Cdc20 and Cdh1, which are encoded by all known eukaryotic genomes [12]. The APC/C ${ }^{\mathrm{Cdc} 20}$ activates the APC/C during early mitosis to promote the cell cycle from metaphase to anaphase, whereas Cdh1 activity is low due to its Cdk-dependent phosphorylation. The APC/ $\mathrm{C}^{\mathrm{Cdh} 1}$ regulates the exit from mitosis $[13,14]$. The $\mathrm{APC} 10$ is the processivity factor of the $\mathrm{APC} / \mathrm{C}$, which promotes the substrates recognition by the destruction box [15]. Research suggested that APC10 stimulates processivity by limiting substrate dissociation, which enforces the ubiquitination stability [16].

This study demonstrated that APC10 interacts with NLRP3 to promote the assembly and the activation of the NLRP3 inflammasome. Notably, APC10 binds to NLRP3 tightly during interphase, whereas in mitosis, APC10 participates in APC/C assembly and disassociates from the NLRP3 inflammasome to release the activation of the NLRP3 inflammasome. Overall, we discovered a distinct mechanism by which APC10 orchestrates the activation of the NLRP3 inflammasome during cell cycle control.

\section{Results}

\subsection{APC10 protein interacts directly with NLRP3 protein}

In order to reveal the molecular mechanism underlying the regulation of the NLRP3 inflammasome activation, we initially screened cellular proteins interacting with NLRP3 PYD domain using yeast two-hybrid system. The interaction was verified by co-immunoprecipitation (CoIP) in human embryonic kidney (HEK293T) cells. Co-IP results showed that APC10 interacted with NLRP3 (Figure 1A) and NLRP3 interacted with APC10 (Figure 1B). We also noted that endogenous APC10 interacted strongly with endogenous NLRP3 in mouse mononuclear/macrophage (J774A.1) cells (Figure 1C). Yeast two-hybrid results showed that APC10 interact with NLRP3 PYD domain directly (Figure 1D). And APC10 interacted with NLRP3 and its all domains LRR, NAD, and PYD (Figure 1E). Moreover, confocal microscopy demonstrated that NLRP3 and APC10 were colocalized and mainly distributed in cytoplasm (Figure 1F and Figure 1G). Collectively, these results demonstrated that APC10 protein interacts directly with NLRP3 protein. 
A

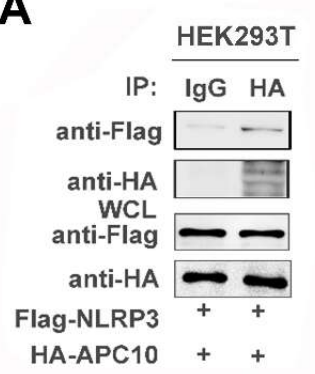

B

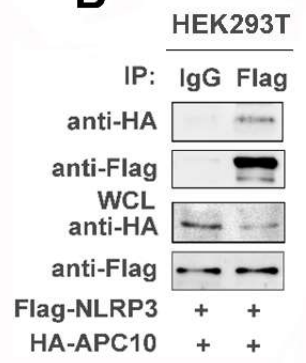

D

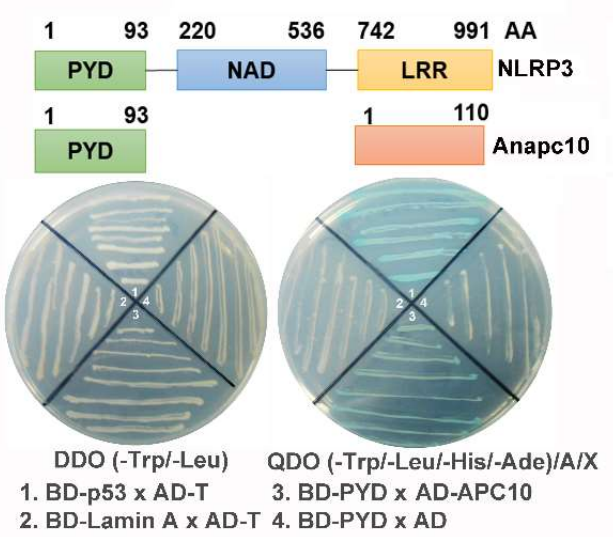

C J774A.1

IP: IgG NLRP3

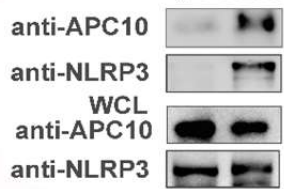

anti-NLRP3

E
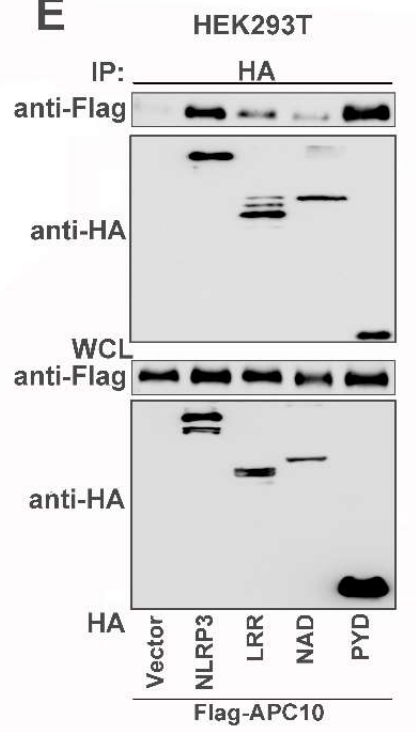

F
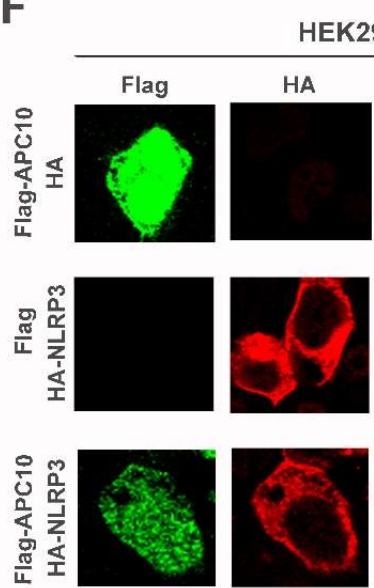
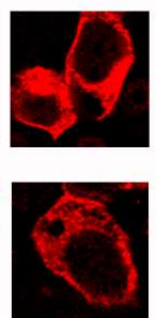

HEK293T
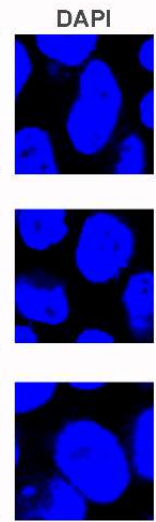
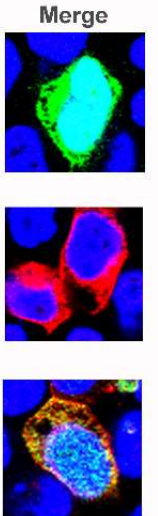

G

Hela
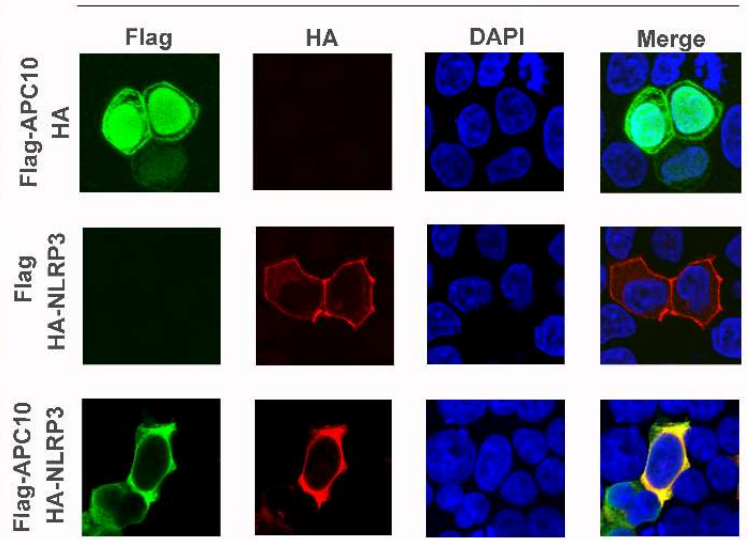

Figure 1. NLRP3 interacts with APC10 through the LRR, NAD and PYD domains. (A and B) HEK293T cells were transfected with HA-APC10 plasmid and Flag-NLRP3 plasmid. Lysates were either analyzed directly by immunoblotting using anti-Flag and anti-HA antibody (WCL) or immunoprecipitated (IP) using control IgG and anti-Flag antibody (A) or control IgG and anti-HA antibody (B) and then analyzed by immunoblotting using anti-Flag and anti-HA antibody. (C) J774A.1 cell lysates were either analyzed directly by immunoblotting using anti-NLRP3 ana anti-APC10 antibody (WCL) or immunoprecipitated (IP) using control IgG and anti-NLRP3 antibody and then analyzed by immunoblotting using anti-NLRP3 and antiAPC10 antibody. (D) Yeast strain Y2HGold was cotransformed with combination of binding domain (BD) and activation domain $(\mathrm{AD})$ plasmid. Transfected yeast cells were grown on SD-minus Trp/Leu double dropout (DDO) plates, and colonies were transfered onto SD-minus Trp/Leu/Ade/His plates containing 
Aureobasidin A and X- $\alpha$-gal (QDO/A/X). (E) HEK293T cells were transfected with Flag-APC10 plasmid along with plasmids encoding HA-Vector, HA-NLRP3, HA-LRR, HA-NAD, or HA-PYD. Lysates were either analyzed directly by immunoblotting using anti-Flag and anti-HA antibody (WCL) or immunoprecipitated (IP) using anti-HA antibody and then analyzed by immunoblotting using anti-Flag and anti-HA antibody. (F) HEK293 T cells were co-transfected with plasmid encoding Flag-APC10 and plasmid expressing HA-NLRP3. Subcellular localizations of Flag-APC10 (green), HA-NLRP3 (red) and nucleus marker DAPI (blue) were analyzed with Immunofluorescence microscopy. (G) Hela cells were co-transfected with plasmid encoding Flag-APC10 and plasmid expressing HA-NLRP3. Subcellular localizations of FlagAPC10 (green), HA-NLRP3 (red) and nucleus marker DAPI (blue) were analyzed with Immunofluorescence microscopy.

\subsection{Anapc10 promotes the activation of the NLRP3 inflammasome}

The effect of APC10 on the regulation of the NLRP3 inflammasome activity was determined by over-expression of APC10 and knock-down of APC10 in a reconstructed NLRP3 inflammasome cell system. HEK293T cells were co-transfected with plasmids encoding the three components (NLRP3, ASC, and pro-Casp-1) of the NLRP3 inflammasome along with plasmid encoding the substrate proIL-1 $\beta$ to generate a NLRP3 inflammasome cell system as described previously $[17,18]$. The HEK293T-NLRP3 inflammasome system cells were transfected with plasmid encoding APC10. The results showed that the secretion of mature IL-1 $\beta$ in the supernatants was significantly enhanced by APC10 (Figure 2A) and the production of mature IL-1 $\beta$ (p17) was facilitated in the presence of APC10 (Figure 2B). In addition, the HEK293T-NLRP3 inflammasome system cells were transfected with shAPC10 (a shRNA specific to APC10). Notably, the secretion of mature IL-1 $\beta$ in the supernatants was significantly repressed in the presence of shAPC10 (Figure 2C) and the production of mature IL-1 $\beta$ (p17) was suppressed in the presence of shAPC10 (Figure 2D). Collectively, these results demonstrated that over-expression of APC10 leads to promoting the NLRP3 inflammasome activation, and knock-down of APC10 results in attenuating the NLRP3 inflammasome activation, and therefore, APC10 plays a positive role in promoting the NLRP3 inflammasome activation.

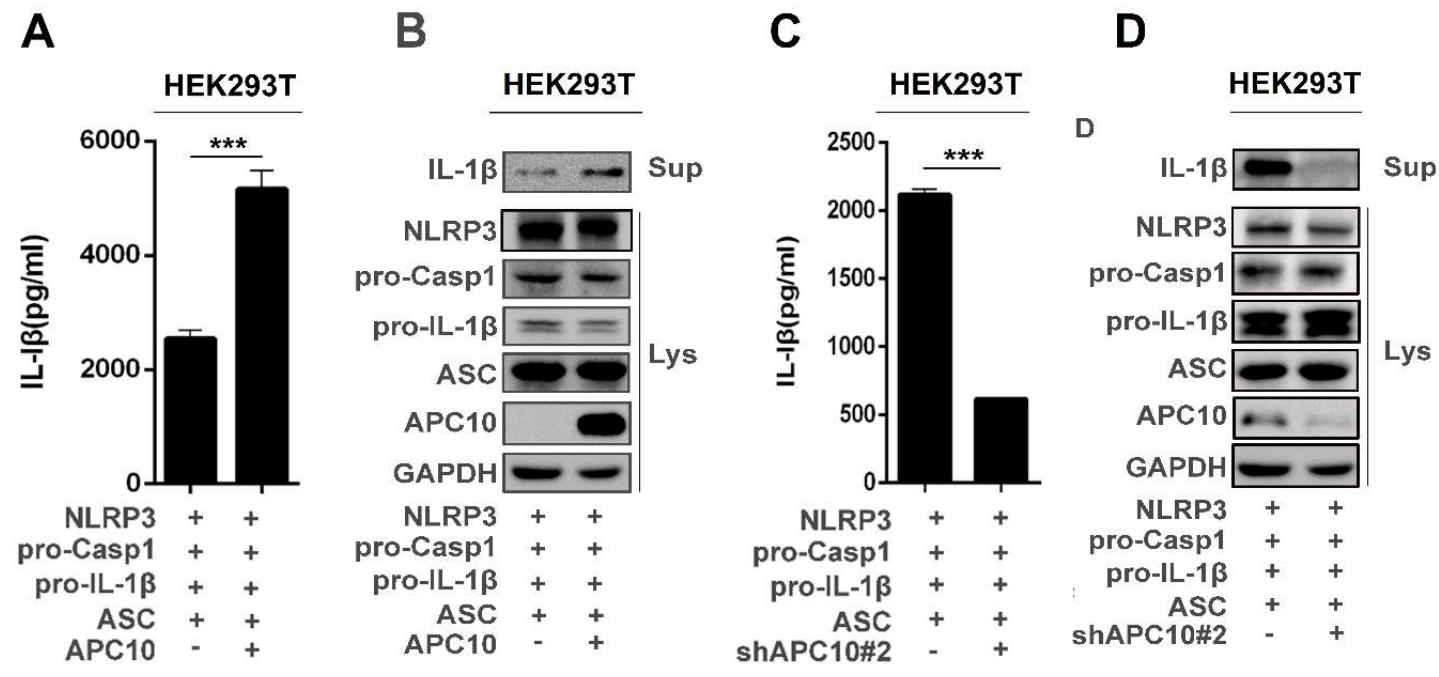

Figure 2. APC10 promotes the activation of NLRP3 inflammasome in HEK293T cells. (A and B)

HEK293T cells were transfected with plasmids encoding Flag-NLRP3, Flag-pro-IL- 1 $\beta$, Flag-pro-caspase-1, 
Flag-ASC and with (+) or without (-) APC10. Supernatants of the cell cultures were analyzed by ELISA for the detection of IL-1 $\beta$ secretion (A). Cell lysates were normalized for protein content and then analyzed by immunoblotting using antibodies for IL-1 $\beta$, NLRP3, Caspase-1, ASC, APC10, and GAPDH (B). (C and D) HEK293T cells were transfected with plasmids encoding Flag-NLRP3, Flag-pro-IL-1 $\beta$, Flag-pro-caspase-1, Flag-ASC, with (+) or without (-) shAPC10\#2. Supernatants of the cell cultures were analyzed by ELISA for the detection of IL-1 $\beta$ secretion (C). Cell lysates were normalized for protein content and then analyzed by immunoblotting using antibodies for IL-1 $\beta$, NLRP3, Caspase-1, ASC, APC10, and GAPDH (D). Data shown are means $\pm \mathrm{SD}, * \mathrm{p}<0.05, * * \mathrm{p}<0.01, * * * \mathrm{p}<0.0001$.

\subsection{Lack of Anapc10 inhibits the endogenous NLRP3 inflammasome activation in macrophage}

Next, the role of endogenous APC10 in regulating the NLRP3 inflammasome activation was further evaluated in mice (J774A.1) cells transfected with siRNA specific targeting APC10 (siAnapc10). Initially, we generated three siRNAs target mouse APC10, mouse siAPC10\#1, mouse siAPC10\#2, mouse siAPC10\#3, and mouse siNC (as a negative control). The results showed that the most effective condition for the knock-down the endogenous APC10 expression was transfecting with siAPC10\#3 at $50 \mathrm{nM}$ (Figure 3A-C).

Next, mice J774A.1 cells were transfected with siNC or siAPC10\#3 and then primed with LPS and treated with Nigericin. We noted that IL-1 $\beta$ secretion was induced by Nigericin and Nigericininduced IL-1 $\beta$ secretion was attenuated in the presence of siAPC10\#3 (Figure 3D). TNF- $\alpha$ was also induced by LPS, however, LPS-induced TNF- $\alpha$ was relatively unaffected by siAPC10\#3 (Figure $3 \mathrm{E})$. These results suggested that APC10 specifically promotes IL-1 $\beta$ activation and secretion.

The role of APC10 in regulating the NLRP3 inflammasome activation was further investigated in THP-1 cells. Lentivirus expressing three pairs of shRNA specific targeting APC10, shAPC10\#1, shAPC10\#2, and shAPC10\#3, as well as shNC (as a negative control) were generated, respectively. THP-1 cells were transduced with the lentiviruses to generate THP-1 cells stably expressing shNC, shAPC10\#1, shAPC10\#2, and shAPC10\#3, respectively. We noted that the level of Anapc10 protein was significantly attenuated by shAPC10\#2 (Figure 3G), indicating that shAPC10\#2 is the most effective shRNA in the knock-down of APC10. Next, THP-1 cells were transduced with the lentiviruses to generate THP-1 cells stably expressing shNC or shAPC10\#2. The stable cells were differentiated into macrophages, which were then primed with LPS and treated with Alum or Nigericin. The results showed that the secretion of IL- $1 \beta$ in the cell supernatant was induced by Alum or Nigericin, whereas Alum- or Nigericin-induced IL-1 $\beta$ secretion was repressed by shAPC10\#2 (Figure 3H). Similarly, the productions of IL-1 $\beta$ (p17) and Caspase-1 (p20) were induced by Alum or Nigericin, whereas Alum- or Nigericin-induced mature IL-1 $\beta$ (p17) and mature Caspase-1 (p20) productions were repressed by shAPC10\#2 (Figure 3I). These results confirmed that APC10 promotes the production and secretion of mature IL-1 $\beta$ and mature Caspase- 1 , and thus playing a critical role in the activation of the NLRP3 inflammasome. 
A

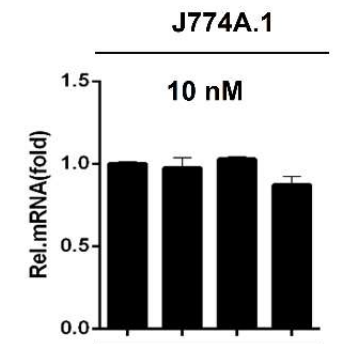

anti-APC10

GAPDH

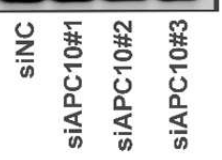

D

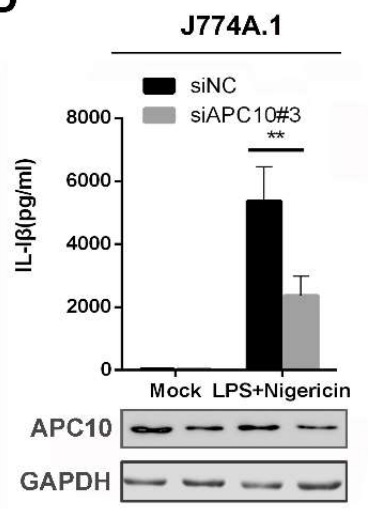

F

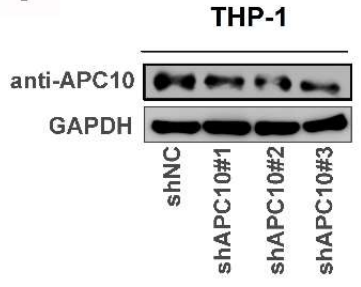

B

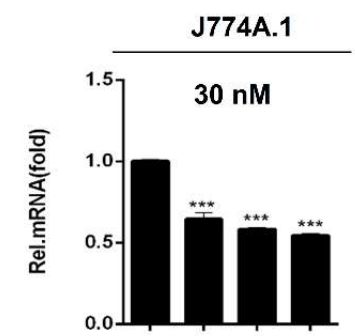

anti-APC10 $m \ldots$

GAPDH

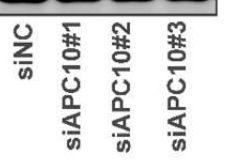

E

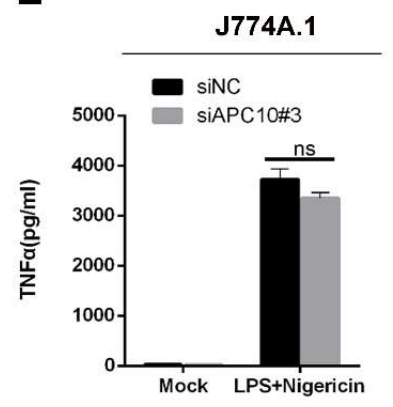

C

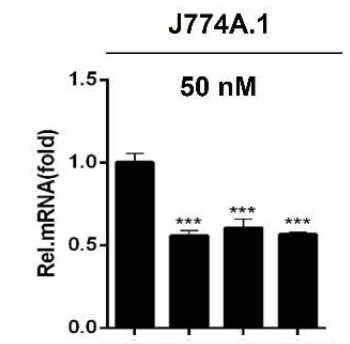

anti-APC10

GAPDH

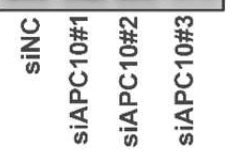

G

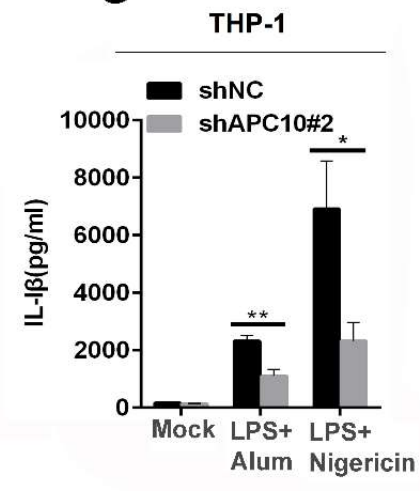

$\mathbf{H}$

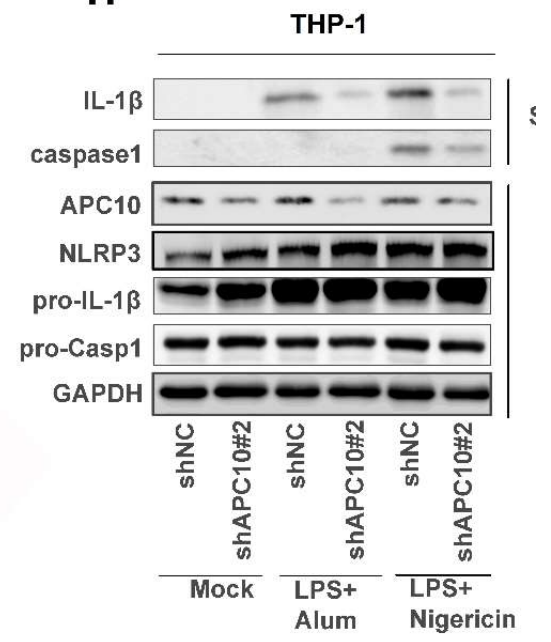

Figure 3. Lack of APC10 will inhibits the activation of NLRP3 inflammasomes in macrophages. (A-C) J774A. 1 cells were transfected with siNC, siAPC10\#1, siAPC10\#2, or siAPC10\#3 with $10 \mathrm{nM}(\mathrm{A}), 30 \mathrm{nM}$ (B), and $50 \mathrm{nM}(\mathrm{C})$. APC10 and GAPDH mRNAs were determined by quantitative RT-PCR (top), APC10 and GAPDH proteins were detected by Western blot analyses (bottom). (D-F) J774A.1 cells were transfected with mouse siNC $(50 \mathrm{nM})$ or siAPC10\#3 $(50 \mathrm{nM})$ which were treated with LPS for $6 \mathrm{~h}$ and Nigericin for $1 \mathrm{~h}$. Supernatants of the cell cultures were analyzed by ELISA for the detection of IL- $1 \beta$ secretion (D) and TNF- $\alpha$ secretion $(E)$. Cell lysates were normalized for protein content and then analyzed by immunoblotting using antibodies for APC10 and GAPDH (F). (G) THP-1 cells were transduced with lentiviruses stably expressing 
shRNA (shNC) and shRNA against Anapc10 (shAPC10\#1, shAPC10\#2, and shAPC10\#3) and selected with puromycin for 2 wks. APC10 and GAPDH proteins were detected Western blot analyses. (H and I) TPAdifferentiated THP-1 cells stably expressing shNC or shAPC10\#2 were treated with LPS for $6 \mathrm{~h}$ and Alum $(400 \mu \mathrm{g} / \mathrm{ml})$ for $6 \mathrm{~h}$, or LPS for $6 \mathrm{~h}$ and Nigericin $(10 \mu \mathrm{M})$ for $1 \mathrm{~h}$. Supernatants of the cell cultures were analyzed by ELISA for the detection of IL-1 $\beta$ secretion $(\mathrm{H})$. Cell lysates were normalized for protein content and then analyzed by immunoblotting using antibodies for IL-1 $\beta$, Caspase-1, APC10, NLRP3, and GAPDH proteins (I). Data shown are means $\pm \mathrm{SD},{ }^{*} \mathrm{p}<0.05, * * \mathrm{p}<0.01, * * * \mathrm{p}<0.0001$.

\subsection{APC10 promotes the assembly of the NLRP3 inflammasome}

NLRP3 forms inactive preassembled complex under normal conditions, whereas it undergoes conformational changes to form active complex in association with ASC and pro-Caspase-1 upon stimulations. Next, the mechanism by which APC10 promotes the NLRP3 inflammasome activation was assessed. HEK293T cells were transfected with pFlag-APC10 along with pHA-vector, pHANLRP3, pHA-ASC, or pHA-Caspase-1. Co-IP results showed that APC10 interacts with NLRP3, ASC, and pro-Caspase-1 (Figure 4A), suggesting that APC10 is tightly associated with the NLRP3 inflammasome. In addition, HEK293T cells were co-transfected with pFlag-NLRP3 and pHA-ASC along with or without pFlag-APC10. Co-IP analyses indicated that APC10 promotes the interaction between ASC and NLRP3 (Figure 4B).

In addition, THP-1 cells were transduced with the lentivirus-shNC and lentivirus-shAPC10\#2 to generate THP-1 cells stably expressing shNC or shAPC10\#2. The stable cells were differentiated in to macrophages, which were then primed with LPS and treated with Nigericin. We noted that ASC oligomerization was induced by Nigericin stimulation, whereas Nigericin-induced ASC oligomerization was attenuated by shAPC10\#2 (Figure 4C). Immunofluorescence microscopy further confirmed that ASC oligomerization was induced by Nigericin stimulation, but Nigericin-induced ASC oligomerization was reduced by shAPC10\#2 (Figure 4D, E). Collectively, these data demonstrated that APC10 interacts with the components of the NLRP3 inflammasome to promote the assembly of the inflammasome complex. 

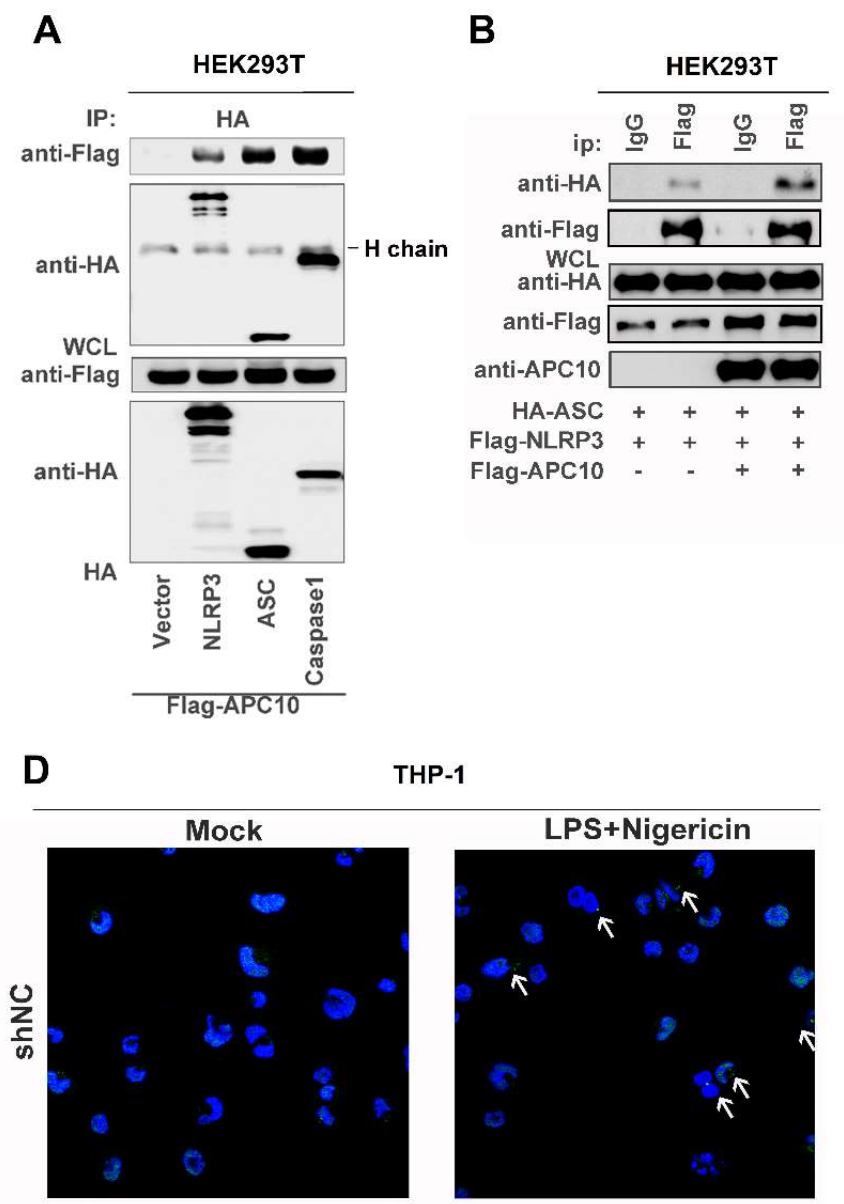

THP-1
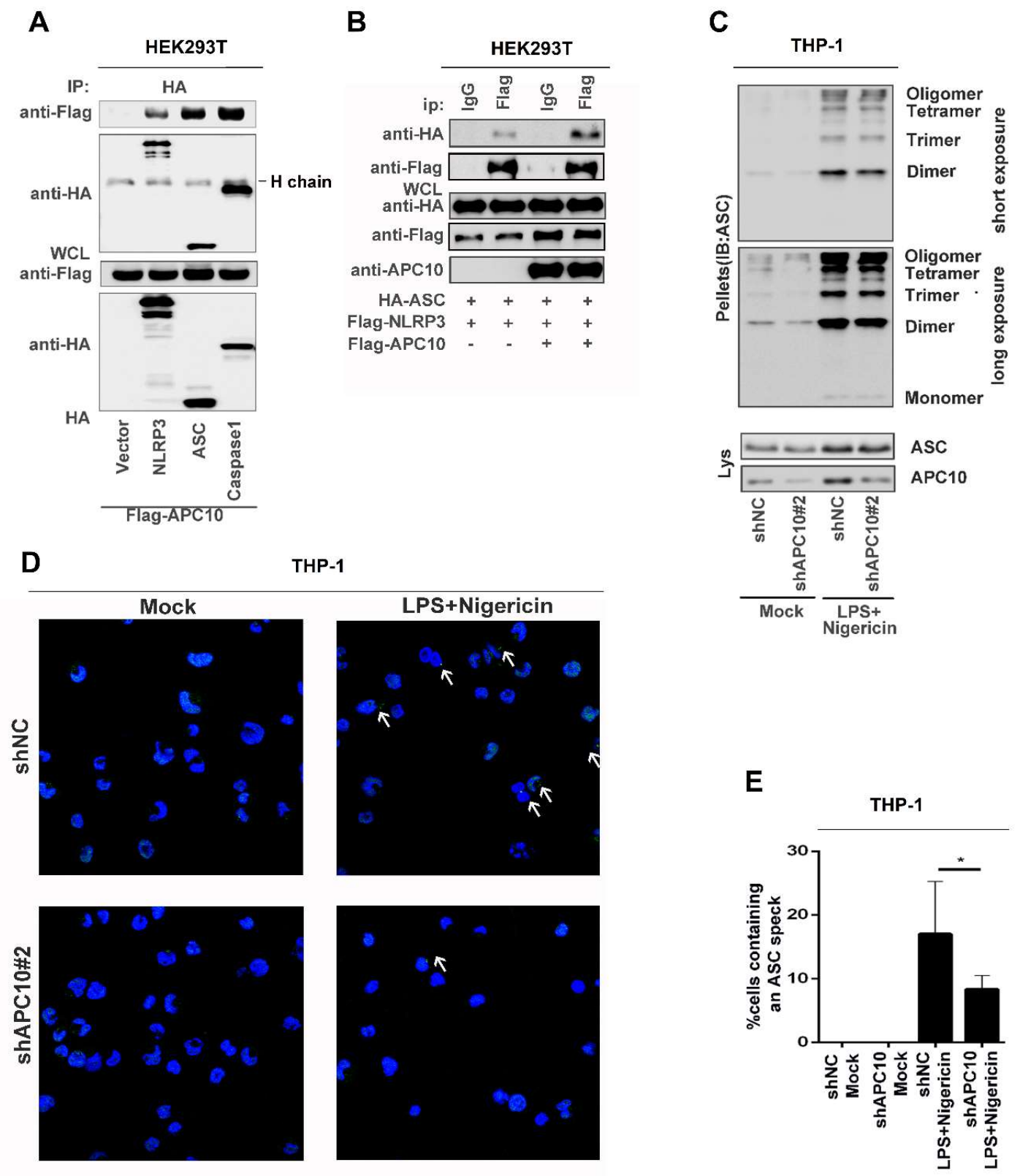

Figure 4. APC10 interacts with NLRP3 inflammasome to promote its assembly. (A) HEK293T cells were transfected with Flag-APC10 plasmid along with plasmids encoding HA-Vector, HA-NLRP3, HA-ASC, or HA-Caspase-1. Lysates were either analyzed directly by immunoblotting using anti-Flag and anti-HA antibody (WCL) or immunoprecipitated (IP) using anti-HA antibody and then analyzed by immunoblotting using anti-Flag and anti-HA antibody. (B) HEK293T cells were transfected with plasmids encoding HA-ASC, Flag-NLRP3 and with (+) or without (-) APC10. Lysates were either analyzed directly by immunoblotting using anti-APC10, anti-Flag and anti-HA antibody (WCL) or immunoprecipitated (IP) using control IgG and anti-Flag antibody and then analyzed by immunoblotting using anti-APC10, anti-Flag and anti-HA antibody. (C) ASC oligomerization in TPA-differentiated THP-1 cells stably expressing shNC or shAPC10\#2 which were treated with LPS for $6 \mathrm{~h}$ and $10 \mu \mathrm{M}$ Nigericin for $1 \mathrm{~h}$. (D and E) TPA-differentiated THP-1 cells stably expressing shNC or shAPC10\#2 which were treated with LPS for $6 \mathrm{~h}$ and $10 \mu \mathrm{M}$ Nigericin for $1 \mathrm{~h}$.

Representative confocal immunofluorescence images (D) and quantification of endogenous ASC specks (E). 
After stimulation, cells were fixed and stained for ASC (Green) and nucleus marker DAPI (Blue). Data shown represent results from three combined independent experiments in which more than 100 cells were counted in each experiment. Data shown are means $\pm \mathrm{SD}, * \mathrm{p}<0.05$.

\subsection{The NLRP3 inflammasome activation is blocked during mitosis}

As the NLRP3 inflammasome activation is strictly controlled during mitosis [19], we thus investigated the effect of APC10 on the NLRP3 inflammasome activation during mitosis and interphase of the cell cycle. Chemical arrest by double-thymidine block (DTB) was used to synchronize J774A.1 cells at the G2/M phase border and released to synchronously enter different phases of cell cycle (Figure 5A). Notably, the highest intensity of the APC10/NLRP3 interaction was detected at $2 \mathrm{~h}$ of DTB release or at interphase and then gradually decreased as mitotic cells increased from $10 \mathrm{~h}$ to $20 \mathrm{~h}$ of DTB release or at mitosis (Figure 5B), indicating that a dynamic interaction between APC10 and NLRP3 was occurred during different cell cycle phases. Moreover, we noted that after LPS and Nigericin treatments, the level of secretion of IL- $1 \beta$ in synchronized mitotic cells was lower than that in unsynchronized cells (Figure 5C). These results suggested that APC10-NLRP3 complex exits in the interphase, which may promote the NLRP3 inflammasome activation. 
A

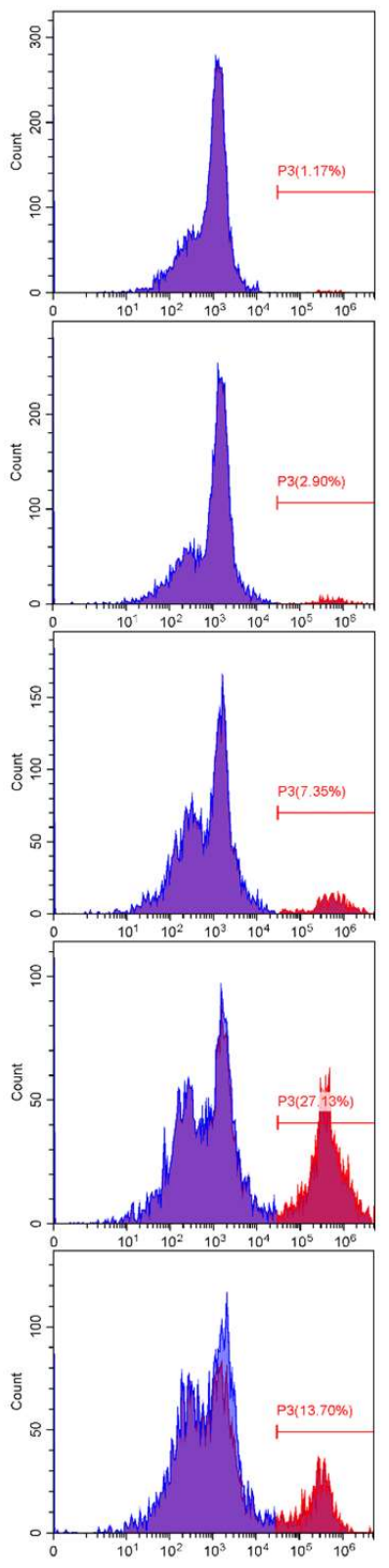

B

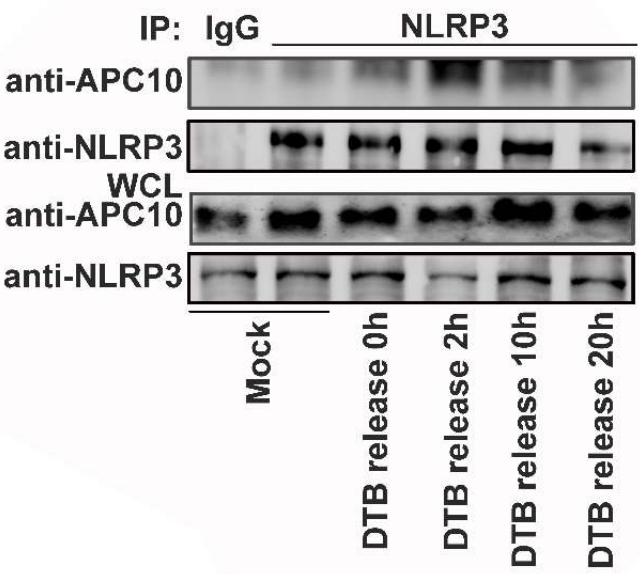

C

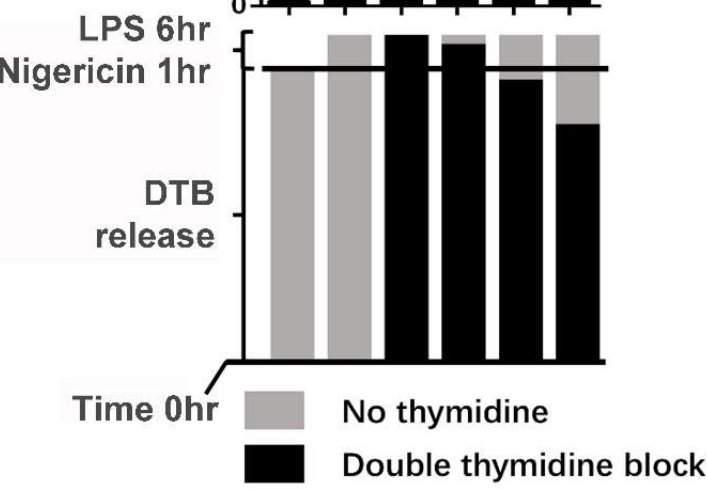

Figure 5. NLRP3 inflammasome activation is inhibited during mitosis. (A-C) J774A.1 cells were arrested at the G2/M phase by treating with double-thymidine block (DTB), then release from arrest for different time periods before analysis. J774A.1 cells were treated with DTB release and were harvested at indicated times (A and B). Flow cytometric analysis of phosphorylated histone H3-positive mitotic cells (A). Lysates were either analyzed directly by immunoblotting using anti-APC10 and anti-NLRP3 antibody (WCL) or immunoprecipitated (IP) using control IgG and anti-NLRP3 antibody and then analyzed by immunoblotting using anti-APC10 anti-NLRP3 antibody (B). ELISA analysis of IL-1 $\beta$ in the culture supernatants of J774A.1 cells primed with LPS and stimulated with nigericin (C). Release from DTB arrest at indicated times (grey) or concurrent with nigericin stimulation (black). The first bar was the supernants of J774A.1 cells without any treatments. The 2-6 bars were the supernants of J774A.1 cells stimulated with LPS for 6 hours and Nigericin for 1 hour. The second bar was the supernants of J774A. 1 cells cultured without double thymidine block. The 
third bar was the supernants of J774A.1 cells blocked by double thymidine release continuously. The 4-6 bars were the supernants of J774A.1 cells blocked by DTB release, and final release from G1 arrest into normal growth medium for 2 hours, 10 hours, and 20 hours respectively. Data shown are means $\pm \mathrm{SD},{ }^{*} \mathrm{p}<0.05$.

Taken together, we discover that APC10 is a critical regulator of the NLRP3 inflammasome activation. APC10 protein interacts and co-localizes with NLRP3 protein in the cytoplasm. During interphase, APC10 interacts with NLRP3 to promote the NLRP3 inflammasome activation, whereas during mitosis, APC10 disassociates from the NLRP3 inflammasome, and thus releasing the inflammatory responses (Figure 6).
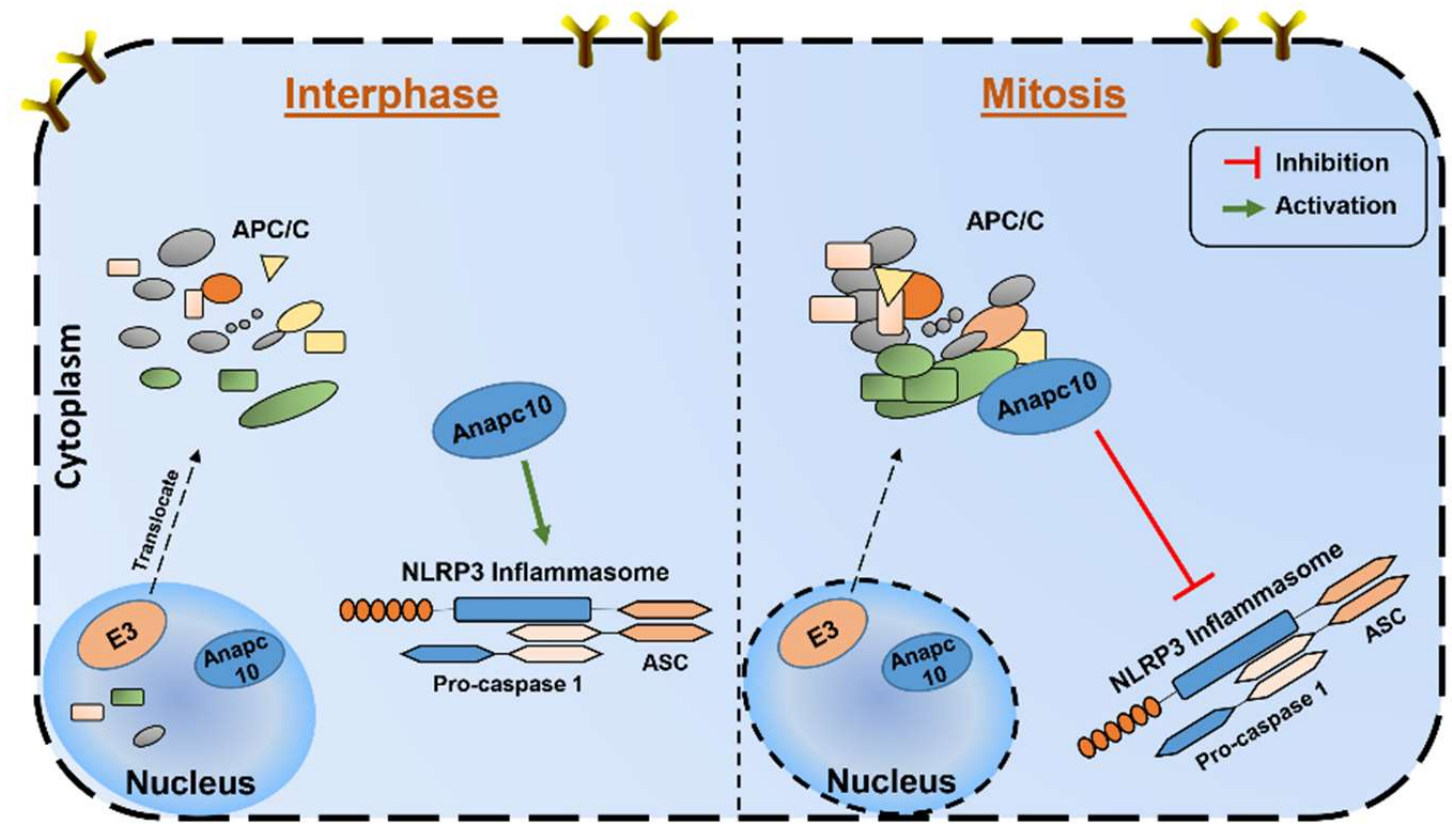

Figure 6. A hypothetical mechanism clarifying the Anapc10 mediates the NLRP3 inflammasome activation during cell cycle. During the interphase, APC10 directly binds with NLRP3 inflammasome to facilitate the assembly of NLRP3 inflammasome complex, which finally promotes the production and secretion of IL- $1 \beta$ and Caspase- 1 . When cell start mitotic division, the APC/C start to assembly, leading to the disparate of APC10 from NLRP3 inflammasome complex, which eventually inhibits the NLRP3 inflammasome activation.

\section{Discussion}

Cell cycle requires the coordinated post-translational modification of the mitotic regulators like Securin and Cyclins, in which APC/C plays an irreplaceable role as an E3 ubiquitin ligase. In this study, we revealed that APC10 interacts with NLRP3 with different approaches including Co-IP and Immunofluorescence microscopy, revealing that APC/C complex and NLRP3 inflammasome complex mutual correlations. Substrates of the APC/C generally contain conserved sequence elements called the destruction box (D-box) and KEN-box [20]. Through the protein comparison, we 
found there is no D-box or KEN-box in NLRP3, ASC and Caspase-1. To our knowledge, there was no report describing a direct interaction between APC10 and its substrate without D-box and KENbox. Moreover, $\mathrm{Cdc} 20$ and $\mathrm{Cdh} 1$ were co-activators of the $\mathrm{APC} / \mathrm{C}$, so that we postulate that APC10 might recruit Cdc20 or Cdh1 to NLRP3, which need to be investigated in the future. The former study demonstrated that the carboxy terminus of APC 10 anchors APC 10 to the APC/C while the other region is required for the APC10 to enhance substrate binding to the APC/C [15]. To further demonstrate the APC10 function in NLRP3 inflammasome activation, we need to construct the truncation of APC10 to verify which domain binds to NLRP3. Interaction between NLRP3 and ASC is critical process of inflammasome assembly [21,22]. The interaction of NLRP3 and ASC is promoted by APC10, indicating that APC10 enhances inflammasome assembly. In APC10 knockdown THP-1 cells, the induction of ASC oligomer was attenuated after Nigericin stimulation. Taken together, we demonstrate that APC10 promotes the assembly and activation of the NLRP3 inflammasome.

Due to APC/C is a multi-subunits E3 ligase. Here, we conducted several experiments to demonstrate whether APC10 increases or decreases the ubiquitination of NLRP3. However, the results showed that APC10 had no influence on the ubiquitination of NLRP3. Cell cycle is a complex process that involves many regulatory proteins to guide the cell to product two daughter cells. The center of cell cycle are the cyclin-dependent kinases (CDKs), and cyclin proteins [23]. During mitosis, the nucleus membrane dissociation, the Golgi and ER membrane restructure. These processes may inhibit NLRP3 inflammasome activation, because the induction of IL-1 $\beta$ can induce the production of reactive oxygen species (ROS), which is capable of damaging DNA [24]. Activation of the NLRP3 inflammasome needs to be strictly controlled during mitosis. With the percentage of mitosis cells growth, the interaction between APC10 and NLRP3 decrease. Previous research showed that Cdc20 was localized in cell nucleus [25], Cdh1 located in cytoplasm and Golgi apparatus [26], and NLRP3 was thought localized in cytoplasm [27]. We speculate that due to the switch of Cdc20 and Cdh1, the substrate binding to APC/C is also changed. During mitosis, the $\mathrm{APC} / \mathrm{C}^{\mathrm{Cdc} 20}$ shows low affinity to NLRP3, the interaction between APC10 and NLRP3 is reduced. Moreover, previous study showed that NIMA-related kinases 7 (NEK7), a serine/threonine kinase previously implicated in mitosis also acts downstream of potassium efflux to regulates NLRP3 inflammasome activation [28]. NEK7 binds to NLRP3 directly and the interaction is necessary for NLRP3-ASC formation, ASC oligomerization, and Caspase-1 activation [18]. We propose that NEK7 interacts with APC10, which clarify the mutual influence of cell cycle and inflammasomes completely.

In summary, we discovered a distinct mechanism to control NLRP3 inflammasome, which involved in APC10 as a switch of both cell cycle and inflammatory responses. In interphase cells, APC10 binds to NLRP3 and enhances its assembly to promote NLRP3 inflammasome activation. When cell start to division, APC10 disassociates from NLRP3 inflammasome to repress its activation to keep cell maintenance.

\section{Materials and Methods}

\subsection{Cell lines and cultures}

Human embryonic kidney cell line (HEK293T) and Hela cells was purchased from American Type Culture Collection. Mouse mononuclear/macrophage J774A.1 cell line was purchased from China Center for Type Culture Collection. Human monocytic cell line (THP-1) was a gift from Dr. 
Bing Sun of Institute of Biochemistry and Cell Biology, Shanghai Institute for Biologic Sciences, Shanghai, China. J774A.1 cells, Hela cells and HEK293T cells were maintained in DMEM purchased from Thermo Fisher Scientific supplemented with $10 \%$ fetal bovine serum (FBS), 100 $\mathrm{U} / \mathrm{ml}$ penicillin, and $100 \mathrm{mg} / \mathrm{ml}$ streptomycin sulfate. THP-1 cells were maintained in RPMI 1640 medium supplemented with $10 \% \mathrm{FBS}, 100 \mathrm{U} / \mathrm{ml}$ penicillin, and $100 \mathrm{mg} / \mathrm{ml}$ streptomycin sulfate. Cells were maintained in an incubator at $37^{\circ} \mathrm{C}$ in $5 \% \mathrm{CO}_{2}$.

\subsection{Antibodies and Reagents}

LPS, phorbol-12-myristate-13-acetate (TPA), dansylsarcosine piperidinum salt (DSS), and Thymidine were purchased from Sigma-Aldrich (St Louis, MO, USA). Nigericin and Alum were purchased from InvivoGen Biotech (San Diego, CA, USA). X-a-Gal was purchased from Gold Biotechnology (St. Louis, MO, USA). Aureobasidin A was obtained from Clontech Laboratories (Mountain View, CA, USA).Complete, EDTA-free Protease Inhibitor Cocktail Tablets provided in EASYpacks was purchased from Roche (Basel, Switzerland). Normal rabbit IgG, normal mouse IgG and Lipofectamine 2000 were purchased from Invitrogen Corporation (Carlsbad, CA, USA). PepMute siRNA Transfection Reagent was purchased from SignaGen Laboratories (Frederick, MD, USA). Fixation/Permeabilization Solution Kit was purchased from BD Biosciences (Franklin Lakes, NJ, USA).

Monoclonal mouse anti-Flag (F3165), polyclonal rabbit anti-HA (H6908), and monoclonal mouse anti-glyceraldehyde 3-phosphate dehydrogenase (GAPDH; G9295) antibodies were purchased from Sigma. Polyclonal Rabbit anti-NLRP3 (15101), polyclonal rabbit anti-APC10 (14807), polyclonal rabbit anti-IL-1 $\beta$ (12703) and polyclonal rabbit anti-Caspase-1 (3866) were purchased from Cell Signaling Technology (Beverly, MA, USA). Monoclonal mouse anti-ASC (sc271054) was purchased from Santa Cruz Biotechnology (Santa Cruz, CA, USA). Cy3-conjugate donkey anti-rabbit IgG (A22220) and FITC-conjugate donkey anti-mouse IgG (A22120) antibodies were purchased from Abbkine (California, USA). Anti-Histone H3-phosphorylated (Ser28) antibody (641006) and Rat IgG2a, $\kappa$ Isotype Ctrl antibody (400526) were purchased from BioLegend (San Diego, CA, USA).

\subsection{Yeast 2-hybrid screening}

Saccharomyces cerevisiae strain Y2HGold, control vectors pGBKT7, pGADT7, pGBKT7-p53, pGBKT7- Lamin A, pGADT7-T, and some reagents were purchased from Clontech Laboratories. All experimental procedures were done following the Matchmaker Gold Yeast 2-Hybrid System User Manual.

\subsection{Western blot analysis}

For Western blot analysis, cells were lysed in lyses buffer $(50 \mathrm{mM}$ Tris-HCl, pH7.4, $150 \mathrm{mM}$ $\mathrm{NaCl}, 1 \% \mathrm{NP}-40,5 \mathrm{mM}$ EDTA, and 10\% glycerol). Protein concentration was determined by Bradford assay (Bio-Rad). Cell lysates were separated by 5-12\% SDS-PAGE and then transferred to a nitrocellulose membrane (Millipore Sigma). The membranes were blocked in PBS containing 5\% nonfat dried milk before incubation with specific antibodies. Blots were detected with the Clarity Western ECL substrate (BioRad) and protein bands were detected using a Luminescent Image Analyzer (Fujifilm LAS-4000). 


\subsection{Co-immunoprecipitation assays}

The whole-cell lysates were prepared by lysing cells with buffer $(50 \mathrm{mM}$ Tris-HCl, $\mathrm{pH} 7.5,150$ $\mathrm{mM} \mathrm{NaCl}, 1 \% \mathrm{NP}-40,5 \mathrm{mM}$ EDTA, and 10\% glycerol). Lysates were immunoprecipitated with control mouse immunoglobulin $\mathrm{G}$ ( $\mathrm{IgG}$ ) or indicated primary antibodies at $4{ }^{\circ} \mathrm{C}$ overnight and were incubated with ProteinA/G Sepharose (GE Healthcare) for 2-3 h. The beads were washed 3-5 times by washing buffer ( $50 \mathrm{mM}$ Tris-HCl, pH7.5, $300 \mathrm{mM} \mathrm{NaCl}, 1 \% \mathrm{NP}-40,5 \mathrm{mM}$ EDTA, and 10\% glycerol) and resuspended with the same volume 2 x SDS loading buffer before immunoblot analysis.

\subsection{Quantitative PCR}

Total RNA was extracted with TRIzol reagent (Thermo Fisher Scientific) following the manufacturer's instructions. Real-time quantitative RT-PCR was performed using the Roche LC480 and ChamQ SYBR qPCR Master Mix (Vazyme) in a reaction mixture of $10 \mu$ l ChamQ SYBR qPCR Green MasterMix, $1 \mu \mathrm{l}$ cDNA diluted template, $1 \mu \mathrm{l}$ real-time PCR primers, and RNase free water to complete the $20 \mu \mathrm{l}$ volume. All real-time PCR primers were designed in Nucleotide of National Center for Biotechnology Information (Bethesda, MD, USA). All primers were as follows: GAPDH (Mouse) forward, 5'-AGGTCGGTGTGAACGGATTTG-3', GAPDH (Mouse) reverse, 5'TGTAGACCATGTAGTTGAGGTCA-3'; APC10 (Mouse) forward,5'ATGACCACACCGAACAAGACA-3', APC10 (Mouse) reverse, 5'TCCCGTAATTGATCCACTCCAA-3'.

\subsection{Lentiviral production and infection}

A pLKO.1-encoding short hairpin RNA (shRNA) vector for a scrambled (MilliporeSigma) or a specific-target molecule (MilliporeSigma) was transfected along with pMD2.G (an envelope plasmid) and psPAX2 (a packaging plasmid) into HEK293Tcells. Change fresh culture medium at 12 $\mathrm{h}$ after transfection. HEK293T cells culture supernatants were harvested at $36 \mathrm{~h}$ and $48 \mathrm{~h}$ after transfection. Filtering the culture supernatants through a $0.45 \mu \mathrm{m}$ filter to remove the cells. THP-1 cells and HEK293T cells were infected with collected culture supernatants plus $8 \mu \mathrm{g} / \mathrm{ml}$ Polybrene (Millipore Sigma) for $24 \mathrm{~h}$. Then, $1.5 \mu \mathrm{g} / \mathrm{ml}$ puromycin (Selleck) was added into lentiviral-infected cells for selection. Knockdown efficiency of each shRNA-targeted molecule was identified by RTPCR and immunoblot analysis. The targeting sequences of shRNA for human APC10 are as follows: sh-APC10\#1, 5'-ACAACAGTGAAGACATTATGT-3'; sh-APC10\#2, 5'ACAAGGCATCCGTTATATCTA-3'; and sh-APC10\#3, 5'-CCCTTAACTGACAATCATAAG-3'.

\subsection{RNA interference}

Mouse APC10 siRNA and non-targeting control siRNA were obtained from RioboBio and resuspended in DEPC water at $100 \mu \mathrm{M}$. siRNA were transiently transfected using PepMute siRNA Transfection Reagent according to the manufacturer's protocol. All mouse small interfering RNAs (siRNAs) were synthesized as follows: siM-APC10\#1, 5'-TCAGGGAAATTGGGTCTCA-3'; siMAPC10\#2, 5'-CTCAGCCTCACTTAGTGAA-3'; siM-APC10\#3, 5'CTAGAAACTTACTGGCAAT-3'. 


\subsection{Reconstitution of the NLRP3 inflammasome in HEK293T cells}

HEK293T cells grown at 60-80\% confluence were seeded into 6-cm plates overnight and then transfected with plasmids encoding NLRP3 inflammasome component proteins and pro-IL-1 $\beta$, including $1 \mu \mathrm{g}$ NLRP3, $100 \mu \mathrm{g}$ ASC, $400 \mu \mathrm{g}$ proCaspase- 1 and $1 \mu \mathrm{g}$ pro-IL-1 $\beta$. The cells were washed with culture medium $24 \mathrm{~h}$ after transfection and were incubated for $12 \mathrm{~h}$. Cell pellets were collected and lysed in cell lysis buffer for immunoblot analysis and cell culture medium were collected for ELISA to detect mature IL-1 $\beta$.

\subsection{Immunofluorescence microscopy}

All cells were washed 3 times with PBS, and cells were fixed with 4\% paraformaldehyde for 15 min at room temperature, washed 3 times with PBS, permeabilized with PBS containing $0.5 \%$ Triton X-100 for 5 min, washed 3 times with PBS, and finally blocked with PBS containing 5\% BSA for 1 $\mathrm{h}$ at room temperature. The cells were then incubated with the primary antibody at $4{ }^{\circ} \mathrm{C}$ followed by incubation with FITC-conjugate donkey anti-mouse IgG (Abbkine) and Dylight 649-conjugate donkey anti-rabbit IgG (Abbkine) for $1 \mathrm{~h}$. After they were washed 3 times with PBS, cells were incubated with DAPI (Vector Laboratories) for $5 \mathrm{~min}$ at room temperature, and then washed three more times with PBS. Finally, the cells were analyzed using a confocal laser scanning microscope (FluoView FV 1000; Olympus).

\subsection{Measurement of activated Casp-1 and mature IL-1ß}

One milliliter medium from each well was mixed with $3 \mathrm{ml}$ acetone, vortexed, stored at $-20^{\circ} \mathrm{C}$ for $2 \mathrm{~h}$ and then centrifuged for $5 \mathrm{~min}$ at $340 \mathrm{~g}$. Remove the supernatants, and pellets were dried for 5 min at room temperature. The pellets were dissolved in $50 \mu \mathrm{l} \mathrm{PBS}$ and mixed with SDS loading buffer for western blotting analysis.

\subsection{Cytokine measurements}

The concentrations of human IL- $1 \beta$ in culture supernatants were measured by commercially available ELISA kits (BD Biosciences). The concentrations of mouse IL-1 $\beta$ in culture supernatants were measured by ELISA kits (R\&D Systems). The concentrations of mouse TNF- $\alpha$ in culture supernatants were measured by ELISA kits (R\&D System).

\subsection{ASC oligomerization}

The TPA-differentiated THP-1 cells were lysed by buffer (50 mM Tris, $\mathrm{pH} 7.5,150 \mathrm{mM} \mathrm{NaCl}$, $1 \%$ Nonidetp $40,5 \mathrm{mM}$ EDTA, and 10\% glycerol) at $4{ }^{\circ} \mathrm{C}$. Lysates were centrifugated at $6000 \mathrm{rpm}$ for $15 \mathrm{~min}$. The supernatants of the lysates were mixed with SDS loading buffer for western blot analysis with antibody against ASC. The pellets of the lysates were washed with PBS for three times and cross-linked using fresh DSS ( $2 \mathrm{mM}$, sigma) at $37^{\circ} \mathrm{C}$ for $30 \mathrm{~min}$. The cross-linked pellets were then spanned down and mixed with SDS loading buffer for western blotting analysis. 


\subsection{Cell synchronization and cell cycle arrest}

J774A.1 cells were synchronized by DTB arrest. Briefly, sub-confluent J774A.1 cells were cultured in the presence of thymidine $(2 \mathrm{mM})$ for $20 \mathrm{~h}$ before washing with PBS three times and change into normal growth medium for $9 \mathrm{~h}$. Cells were then cultured in the presence of thymidine ( 2 $\mathrm{mM}$ ) for another $20 \mathrm{~h}$ before final release from G1 arrest into normal growth medium. Cells were then collected at indicated times for subsequent analysis.

Cells were collected and washed by PBS containing 2\%FBS for three times, and then fixed in Fixation/Permeanilization solution (BD Biosciences) for 20min at room temperature. After washed by PBS containing $2 \%$ FBS for two times, stained with Alexa Fluor 647-conjugated phosphorylated (Ser28) histone H3 antibody in $100 \mu 12 \%$ FBS PBS. Cells were analyzed by flow cytometry and further analysis.

\subsection{Statistics}

All experiments were repeated at least 3 times with similar results. All results were expressed as the mean \pm SD. Statistical analysis was carried out using the unpaired, two-tailed Student's $t$ test for 2 groups and 1-way ANOVA for multiple groups (Prism5; GraphPad Software).

\section{Acknowledgements}

We thank Dr. Bing Sun of Institute of Biochemistry and Cell Biology, Shanghai Institute for Biological Sciences, Chinese Academy of Sciences, China, for kindly providing the THP-1 cell line. We also thank Dr. Jiahuai Han of State Key Laboratory of Cellular Stress Biology, School of Life Sciences, Xiamen University, Fujian, China, for kindly providing the cDNA of genes Anapc10.

\section{Funding}

This work was supported by This work was supported by National Natural Science Foundation of China (81730061) and Guangdong Province "Pearl River Talent Plan" Innovation and Entrepreneurship Team Project (2017ZT07Y580).

\section{Author Contributions}

S.H., P.W, W.L., K.W., and J.W. contributed to conception and design research; S.H., Q.X., G.Y., M.A.S., and P.P. contributed to execution and analysis of data; S.H., P.W, S.H., Q.X., G.Y., M.A.S., and P.P.

contributed to perform research; S.H., P.W, M.A.S., and P.P. contributed new reagents or analytic tools; S.H., P.W, and K.W. contributed to drafting the article; J.W. revised the manuscript. All authors have read and approved the final version of the manuscript.

Competing interests: The authors declare no competing interests.

Data and materials availability: All data needed to evaluate the conclusions in the paper are present in the paper. 


\section{References}

1. Meylan, E.; Tschopp, J.; Karin, M., Intracellular pattern recognition receptors in the host response. Nature 2006, 442 (7098), 39-44.

2. Takeuchi, O.; Akira, S., Pattern recognition receptors and inflammation. Cell 2010, 140 (6), 80520.

3. Sutterwala, F. S.; Ogura, Y.; Szczepanik, M.; Lara-Tejero, M.; Lichtenberger, G. S.; Grant, E. P.; Bertin, J.; Coyle, A. J.; Galan, J. E.; Askenase, P. W.; Flavell, R. A., Critical role for NALP3/CIAS1/Cryopyrin in innate and adaptive immunity through its regulation of caspase1. Immunity 2006, 24 (3), 317-27.

4. Schroder, K.; Tschopp, J., The inflammasomes. Cell 2010, 140 (6), 821-32.

5. Martinon, F.; Agostini, L.; Meylan, E.; Tschopp, J., Identification of bacterial muramyl dipeptide as activator of the NALP3/cryopyrin inflammasome. Curr Biol 2004, 14 (21), 1929-34.

6. Booshehri, L. M.; Hoffman, H. M., CAPS and NLRP3. J Clin Immunol 2019, 39 (3), 277-286.

7. Hoffman, H. M.; Mueller, J. L.; Broide, D. H.; Wanderer, A. A.; Kolodner, R. D., Mutation of a new gene encoding a putative pyrin-like protein causes familial cold autoinflammatory syndrome and Muckle-Wells syndrome. Nat Genet 2001, 29 (3), 301-5.

8. Petrilli, V.; Martinon, F., The inflammasome, autoinflammatory diseases, and gout. Joint Bone Spine 2007, 74 (6), 571-6.

9. Davis, B. K.; Wen, H.; Ting, J. P., The inflammasome NLRs in immunity, inflammation, and associated diseases. Annu Rev Immunol 2011, 29, 707-35.

10. Randall W. King, J.-M. P., Stuart Tugendreich, Mark Rolfe, Philip Hieter, and Marc W.

Kirschner. A 20s complex containing cdc27 and cdc16 catalyzes the mitosis-specific conjugation of ubiquitin to cyclinB. Cell 1995, 81 (2), 279-88.

11. Valery Sudakin, D. G., Aviva Dahan, Hannah Heller, Judith Hershko, Francis C. Luca, Joan V. Ruderman, and Avram Hershko. The cyclosome a large complex containing cyclin selective ubiquitin ligase activity targets cyclins for destruction at the end of mitosis. Mol Biol Cell 1995, 6 (2), 185-97.

12. Peters, J.-M., The Anaphase-Promoting Complex Proteolysis in Mitosis and Beyond. Mol Cell 2002, 9 (5), 931-43.

13. Rosella Visintin, S. P., Angelika Amon. Cdc20 and cdh1 a family of substrate specific activators of apc dependent proteolysis. Science 1997, 278 (5337), 460-3.

14. Peters, J. M., The anaphase promoting complex/cyclosome: a machine designed to destroy. Nat Rev Mol Cell Biol 2006, 7 (9), 644-56.

15. Carroll, C. W.; Enquist-Newman, M.; Morgan, D. O., The APC subunit Doc1 promotes recognition of the substrate destruction box. Curr Biol 2005, 15 (1), 11-8.

16. Carroll, C. W.; Morgan, D. O., The Doc1 subunit is a processivity factor for the anaphasepromoting complex. Nat Cell Biol 2002, 4 (11), 880-7.

17. Wan, P.; Zhang, Q.; Liu, W.; Jia, Y.; Ai, S.; Wang, T.; Wang, W.; Pan, P.; Yang, G.; Xiang, Q.; Huang, S.; Yang, Q.; Zhang, W.; Liu, F.; Tan, Q.; Zhang, W.; Wu, K.; Liu, Y.; Wu, J., Cullin 1 binds and promotes NLRP3 ubiquitination to repress systematic inflammasome activation. The FASEB Journal 2019, 33 (4), 5793-5807.

18. Wang, W.; Xiao, F.; Wan, P.; Pan, P.; Zhang, Y.; Liu, F.; Wu, K.; Liu, Y.; Wu, J., EV71 3D Protein Binds with NLRP3 and Enhances the Assembly of Inflammasome Complex. PLoS Pathog 2017, 13 (1), e1006123.

19. Shi, H.; Wang, Y.; Li, X.; Zhan, X.; Tang, M.; Fina, M.; Su, L.; Pratt, D.; Bu, C.

H.; Hildebrand, S.; Lyon, S.; Scott, L.; Quan, J.; Sun, Q.; Russell, J.; Arnett, S.; Jurek, P.; Chen, D.; Kravchenko, V. V.; Mathison, J. C.; Moresco, E. M. Y.; Monson, N. L.; 
Ulevitch, R. J.; Beutler, B., NLRP3 activation and mitosis are mutually exclusive events coordinated by NEK7, a new inflammasome component. Nature Immunology 2015, 17 (3), 250-258.

20. Cathie M.Pfleger and Marc W.Kirschner., The KEN box: an APC recognition signal distinct from the D box targeted by cdh1. Genes Dev 2000, 14 (6), 655-65.

21. Fernandes-Alnemri, T.; Wu, J.; Yu, J. W.; Datta, P.; Miller, B.; Jankowski, W.; Rosenberg, S.; Zhang, J.; Alnemri, E. S., The pyroptosome: a supramolecular assembly of ASC dimers mediating inflammatory cell death via caspase-1 activation. Cell Death Differ 2007, 14 (9), 1590-604.

22. Proell, M.; Gerlic, M.; Mace, P. D.; Reed, J. C.; Riedl, S. J., The CARD plays a critical role in ASC foci formation and inflammasome signalling. Biochem J 2013, 449 (3), 613-21.

23. K.A.Schafer, The Cell Cycle: A Review. Vet Pathol 1998, 35, 461-78.

24. Bertram, C.; Hass, R., Cellular responses to reactive oxygen species-induced DNA damage and aging. Biol Chem 2008, 389 (3), 211-20.

25. Guttery, D. S.; Ferguson, D. J.; Poulin, B.; Xu, Z.; Straschil, U.; Klop, O.; Solyakov, L.; Sandrini, S. M.; Brady, D.; Nieduszynski, C. A.; Janse, C. J.; Holder, A. A.; Tobin, A. B.; Tewari, R., A putative homologue of CDC20/CDH1 in the malaria parasite is essential for male gamete development. PLoS Pathog 2012, 8 (2), e1002554.

26. Gaudet, P.; Livstone, M. S.; Lewis, S. E.; Thomas, P. D., Phylogenetic-based propagation of functional annotations within the Gene Ontology consortium. Brief Bioinform 2011, 12 (5), 449-62. 27. Subramanian, N.; Natarajan, K.; Clatworthy, M. R.; Wang, Z.; Germain, R. N., The adaptor MAVS promotes NLRP3 mitochondrial localization and inflammasome activation. Cell 2013, 153 (2), 348-61.

28. He, Y.; Zeng, M. Y.; Yang, D.; Motro, B.; Núñez, G., NEK7 is an essential mediator of NLRP3 activation downstream of potassium efflux. Nature 2016, 530 (7590), 354-357. 